\title{
Effect of mobile-based self-management application on stroke outcomes: A study protocol for triple blinded Randomized Clinical Trial
}

Hamidreza Tadayon

Kashan University of Medical Sciences

Mehrdad Farzandipour

Kashan University of Medical Sciences

Ehsan Nabovati

Kashan University of Medical Sciences

Hossein Akbari

Kashan University of Medical Sciences

Seyed Ali Masoud ( $\nabla$ masoud_s_a@yahoo.com )

Kashan University of Medical Sciences https://orcid.org/0000-0001-9038-9838

\section{Research Article}

Keywords: Stroke, Telemedicine, Telerehabilitation, Mobile Applications, Self-Care

Posted Date: May 4th, 2021

DOl: https://doi.org/10.21203/rs.3.rs-284523/v1

License: (9) This work is licensed under a Creative Commons Attribution 4.0 International License. Read Full License 


\section{Abstract}

Background: Stroke is known as the second leading cause of death and the main leading cause of longterm disabilities in the world. Self-care applications can be potentially effective in improving chronic conditions. This protocol will be implemented for a study to evaluate the effects of an Android-based selfcare application on patients with stroke.

Methods: The first stage will include the development of an android-based application and second stage will be conducted using triple blinded Randomized Clinical Trial (RCT). The sample size will include 60 patients with recent stroke and partial paralysis of limbs (hemiplegia or hemiparesis), who will be divided into two groups of intervention and control through permuted block randomization method. Patients in both groups will receive usual medical care, but those in the intervention group will also use an Androidbased self-management application for a period of two months and a total of 40 sessions. Outcomes will be assessed using valid and reliable questionnaires.

Discussion: Assessed outcomes will include stroke severity using National Institute of Health Stroke Scale (NIHSS) score, ability to perform activities of daily living using Barthel Index (BI) score, depression rate using Beck Depression Inventory (BDI-II) score, quality of life using EQ-5D-3L score, medication adherence using Modified Morisky Scale (MMS-8) score, patient satisfaction using Patient Satisfaction Questionnaire (PSQ) score and the number and type of complications in patients into two groups. These outcomes will be assessed at baseline, after two months and after three months from the beginning of the intervention. Intervention effects on the measured variables will also be evaluated using appropriate statistical tests based on the type of variable distribution. Potential consequences of the study might be the improvement of the measured variables in the intervention group compared to the control group. The initial expectations are that the intervention may significantly improve the status of the measured variables in the intervention group compared to that of the control group.

Trial registration: This protocol is registered in the Iranian registration of clinical trial (IRCT) on November 7, 2020 with the code IRCT20201015049037N1. URL: https://irct.ir/trial/51674

\section{Background}

Every year, 26 million people worldwide experience stroke, and the disease is the second leading cause of death worldwide and the main leading cause of long-term disabilities [1]. According to the World Health Organization (WHO), of the 56 million deaths that occur worldwide each year, 10.8\% are due to stroke [2]. Stroke includes a group of pathological disorders of cerebral arteries characterized by decreased neurological and non-convulsive function due to cerebral ischemia or intracranial hemorrhage [3]. Stroke has a variety of consequences for patients [4] and stroke survivors usually experience a wide range of symptoms related to motor function, speech, swallowing, vision, sensation and perception, and their recovery can be slow and incomplete $[5,6]$. These symptoms often lead to limited participation in home and community activities. Rehabilitation procedures for these people are often long-term and require a lot 
of resources. However, only half of survivors have access to rehabilitation services after discharge [7]. In a qualitative study, Anderson et al. [8] concluded that mobile-based applications could improve self-care in patients with chronic conditions. Other studies have shown the impacts of mobile-based training on improving stroke outcomes $[9,10]$. An increasing number of RCTs have evaluated the effectiveness of telerehabilitation interventions. Nevertheless, it is difficult to draw conclusions about the effects of these interventions since the type of interventions and comparisons performed vary greatly from study to study. Some studies have shown the effects of mobile-based applications on improving and enhancing some of the measured outcomes in patients after stroke [11-14] while other studies showed the use of these applications could be ineffective based on the measured outcomes $[15,16]$. In addition, the results of a number of studies do not seem to be generalizable and might be at risk of being bias, and accordingly more studies are needed to yield more definitive conclusions [7]. Therefore, in this study, we intend to first develop a mobile-based application and then solve its usability problems by expert-based usability evaluation. Finally, an interventional study will evaluate the effects of this application on patients with stroke.

The effect assessment of the application will be done by focusing on the three main parts of the ICF, namely the physical structure, individuals' activities and social participation.

\section{Methods}

This Randomized Clinical Trial (RCT) will be done in two stages. The first stage will include the development of an android-based application, with the second stage being the evaluation of the effects of this application on patients with stroke.

\subsection{Stage 1: application development}

Application development will be based on the model presented by Chavez et al. in 2019 [17] (Fig. 1). This model is a combination of iterative and incremental models in system development. According to this model, software development will be done in three different phases, which include the following:

- - Determining functional requirements

- - Designing a low-fidelity prototype and evaluating its validity

- - Designing a High-fidelity prototype and evaluating its usability

\subsubsection{Determining functional requirements}

In this phase, the functional requirements of the application will be determined.

Functional requirements will be obtained through the following four methods: 
- - Selecting clinical guideline

- - Reviewing similar studies and applications

- - Needs assessment of patients

- - Needs assessment and localization of requirements based on expert opinion

In this phase, based on the existing stroke self-care guidelines, one of the clinical guidelines approved by three neurologists will be selected at first and the functional requirements of this guideline will be extracted by researchers.

Other functional requirements will also be extracted using a review study on applications done by researchers and similar studies.

In this regard, some systematic review studies have been conducted $[7,18-21]$ which will also be reviewed by researchers. Needs assessment of patients will be done as the most important stakeholder group of the application. For this purpose, a semi-structured interview will be used with patients who have previously suffered from stroke and partial paralysis and have now partially recovered. These patients are required to have at least a diploma and be able to work with a smartphone. According to similar studies, participants will include three patients $[22,23]$ who will be selected by convenience sampling method. Finally, all these requirements will be prepared in the format of Lawshe Table and will be given to the experts. At this stage, a questionnaire containing functional requirements and information content of the application will be given to neurologists, occupational therapists and physiotherapists for the purpose of localization. Delphi technique will be used in this step. Moreover, for needs analysis, 15-21 neurologists, physiotherapists and occupational therapists who are faculty members of the country's medical universities and have at least three years of professional experience will be consulted. At this stage, for each requirement, the necessity, appropriateness, clarity and relevance will be evaluated and in terms of necessity (CVR), three levels (essential and useful, useful but unnecessary, non-useful) and in terms of appropriateness, clarity and relevance (CVI) four levels (from very high with a score of four to very low with a score of one) will be defined.

Questions with a CVR score of higher than the base number ( 0.62 for ten individuals) based on Lawshe table will be approved and those with a CVR score of less than that will be removed.

According to $\mathrm{CVI}$, questions that have scores of higher than 0.79 will be approved and scores between 0.7 to 0.79 will be re-reviewed, corrected and returned to the experts and finally those of less than 0.7 will be rejected. This step will be repeated until the final validity of the questionnaire content is confirmed.

The approved questionnaire will be provided to the experts in the next step and the experts' answers to the components of the questionnaire will be scored from 1 to 5 (very low $=1$, low $=2$, medium $=3$, high $=4$ and very high $=5$ ) and then the average score of each item will be calculated.

Minimum score for accepting each component will be considered as 3.75 .

If the score obtained for each component is between 2.5 to 3.74 , that component will be modified and reviewed again. 
Components with a score of less than 2.5 will also be removed from the application content.

\subsubsection{Development of low-fidelity interface}

In this phase, based on the feedback and opinions received from experts in the previous step, first the items and how the application works as a conceptual model will be designed and then the low-fidelity user interface (how to display the content of the application and the relationship between humans and smartphones) on android platform will be developed using the appropriate programming language. Then, a primary prototype will be created and its validity will be evaluated. After implementing functional requirements on the prototype for checking validity, six experts will review the application and evaluate the extent to which functional requirements and goals are met by this prototype. These experts will include two neurologists, two physiotherapists and two occupational therapists with at least three years of specialized work experience in the field of treatment and rehabilitation of patients with stroke.

\subsubsection{Development of high-fidelity interface}

At this phase, based on the final version of the prototype obtained from the previous phase, the highfidelity prototype will be developed.

In order to evaluate the usability of the prototype, Cognitive Walkthrough (CW) method will be used based on the method proposed by Polson and Lewis [24]. For this purpose, a certain number of scenarios that represent the most important and frequent application functions will be identified in line with the opinion of three neurologists. For each scenario, the main objective, sub-objectives, sequence of steps to do the tasks and system responses will be prepared and approved by the researchers. Then, the program will be provided to the evaluators. Five individuals will be chosen to do the evaluation through non-probability quota sampling. Evaluators are required to have at least a master's degree in health information technology/management or medical informatics and have 2 years of professional experience in evaluating health information systems. They will independently perform the sequence of steps for each scenario through the program's user interface. Each evaluator puts themselves in the position of the patient and observes the program from the patient's point of view; in case of any problems in performing task steps, it will be expressed by evaluators. This will happen at the presence of the researcher as an observer who will write the following items in data collection forms: evaluator's comments, questions and uncertainties, an explanation of the usability problem identified by the evaluator, location of the problem in the system and the time to perform each scenario. At the end of each evaluation process, each evaluator will review the list of related problems and suggest any solutions they have to solve potential issues. Then, in a meeting, separate lists of evaluators will be compared and all identified problems will be put on the list of main problems. Next, any duplicate problems will be eliminated and the evaluators will be asked to assess the severity of the problems independently. This rating will be based on three criteria including frequency of the problem, impact of the problem and continuity of the problem. Scoring for each problem and criterion will be done in 5 degrees ranging from 0 (no problem) to 4 (severe problem). 
Then, the average of the identified problems will be calculated and relevant problems will be listed in order of intensity followed by feedback given to application designers.

Also, in order to confirm the application by users, the Technology Acceptance Model (TAM) will be used and Perceived Ease Of Use (PEOU), Perceived Usefulness (PU), User Satisfaction (US) and Attribute of Usability (AU) questionnaires (20 items in total) will be distributed among 30 users who have previously had a stroke and have been working with the application for a week. The results of users' comments are also given to the designers. This step will be repeated until the application is approved by evaluators. The output of this phase is the final version of the application which will be ready to be implemented in the live environment.

\subsection{Application testing}

At this stage, the unit test of the final application will be performed by researchers and different modules of the application will be tested separately to confirm correct performance, which can help to eliminate potential any defects. In the next step, application integrity test will be performed by researchers. For this purpose, one researcher will put him/herself in the position of the patient and another researcher in the position of the physician. Feedback on probable defects of the application will be given to application designers.

\subsection{Implementation and evaluation of application in live environment (clinical trial)}

\subsubsection{Study design and setting}

At this stage, an interventional study with patient blinding, assessor physician and analyst will be conducted to evaluate the effects of the application on patient self-care. The designed application will be installed on patients' smartphones and the necessary training to use it will be given to the patient and their caregivers. The study population will include patients referring to one of the general hospitals in Kashan where sampling can be done in a convenient way. Patients with hemiplegia or hemiparesis caused by a recent stroke will be chosen for this purpose.

Either a patient or a patient's caregiver must have an Android-based smartphone and be able to work with it.

At least 72 hours must have passed since the stroke (Acute phase of the disease must has passed) and patients must have adequate consciousness and hearing and be willing to participate in the study.

Exclusion criteria include:

- - Patients who have some levels of consciousness disorder

- - Patients with cognitive disorders such as dementia or memory impairment

- - Patients with global aphasia

The formula 


\section{"n $=\left(\mathrm{Z}_{1-\alpha / 2}+\mathrm{Z}_{1-\beta}\right)^{2} *\left[\left(\mathrm{~S}_{1}\right)^{2}+\left(\mathrm{S}_{2}\right)^{2} /\left(\bar{x}_{1-\bar{x}_{2}}\right)^{2}\right] "$}

with the reliability of $95 \%$ and the test power of $90 \%$ based on the mean and standard deviation obtained in the same study [25] was calculated as 23 patients, which increased to 30 patients according to the available facilities.

Accordingly, two groups each including 30 patients (intervention and control) with stroke (60 patients in total) who meet the inclusion criteria will be selected.

\subsubsection{Randomization}

Randomization will be done by block method (Permuted Block Randomization) in a way that first all foursome blocks which include two codes A and B will be prepared ( 6 blocks) and then, using a random number table, random blocks will be selected by placement (15 blocks). These blocks contain up to 60 codes including $A$ and $B$, each of which is randomly assigned to either the control or the intervention groups.

\subsubsection{Blinding}

For the purpose of blinding, an application will be installed on the smartphones of patients of both intervention and control groups. The program installed for patients in the control group only contains online questionnaires that patients need to complete and submit at specified intervals. Online questionnaires include a number of questionnaires used to measure the target variables.

However, the program installed on the phones of participants in the intervention group will include all the capabilities and requirements of the self-care program designed in the design phase.

Patients will not be familiar with the nature and content of the installed program on opposite group's phones. During program installation, someone other than the assessor physician will install the program for patients or their caregivers. Furthermore, during the assessment process, the same person will record the results of the physician evaluation and the scores related to each patient. Therefore, the assessor physician will not know which group a given patient belongs to. Numeric codes for intervention and control groups will also be used for analysis, and the letters A and B will be used for the control and intervention groups when information is sent to the analyst.

\subsubsection{Intervention}

Intervention for patients in the intervention group includes an Android-based application that has all the functional requirements approved by experts in the analysis and software design phases. This application contains audio, video, textual files or games to improve lower and upper limb function, improve activities of daily living, prevent and reduce depression, reduce and manage pain and other possible complications of stroke, medication reminders and whatever playing a role in improving patient outcomes, and has been designed according to experts' opinion. Patients will be advised to use the application 5 days a week and for a period of 1 hour each day. The duration of the intervention will be 8 
weeks (40 sessions in total). The application can record the number of times and hours of application use by the user. In order to learn how to use the application, users can contact one of the research team members. A member of the research team will also make weekly phone calls with users to make sure patients know how to use the application.

Intervention for patients in the control group includes usual medical care based on the opinion of the responsible neurologist.

An application will be installed on the smartphones of these individuals, which lacks the main capabilities of the application and only contains electronic questionnaires. Installation of this application will be done only for blinding purposes. Intervention duration and the time of measuring the outcomes will be the same for both groups. (Fig. 2 shows the flowchart of protocol for interventional trial).

\subsubsection{Data collection}

The required data will be collected using valid and reliable questionnaires that are introduced as follows. Data will be collected in three stages: prior to intervention (before the intervention and at the time of discharge), two months after the intervention and three months after the intervention (in the doctor's office or in the hospital outpatient clinic). Data from NIHSS and BI questionnaires and stroke complication checklist will be completed by the attending neurologist and other questionnaires will be completed by patients as self-report. Completion of questionnaires by patients will be done electronically and sent online through the application.

\subsubsection{Stroke severity}

The National Institute of Health Stroke Scale (NIHSS) questionnaire will be used to measure stroke severity.

The data obtained from this questionnaire covers the section of body structure related to ICF. This questionnaire has 11 items, each of which has 5 options and scores from 0 to 4 (a score of 0 indicates normal performance and a score of 4 indicates a severe defect in performance). Due to the special type of scoring, scores will eventually be obtained between 0 to 42 , which will be divided as follows:

0: No stroke symptoms

1-4: Mild stroke

5-15: Medium stroke

16-20: Moderate to severe stroke

21-42: Severe stroke 
The validity of this questionnaire was confirmed using clinical predictor validity and its reliability was approved with Cronbach's alpha of 0.95 in Hinkle's study [26]. It should be noted that, in the present study, the original version of this questionnaire will be used by a neurologist.

\subsubsection{Activities of daily living}

The Barthel Index (BI) questionnaire will be used to measure activities of daily living (covering the activities section of ICF). This questionnaire includes 10 key activities of daily living including: feeding, bathing, grooming, dressing, bowels, bladder, toilet use, transfers (bed to chair and back), mobility (on level surfaces) and stairs. In each item, scores are: 0 (dependent), 5 (need for major help), 10 (need for minor help) and 15 (independent). Finally, the ranking of people is done as follows:

80-100: independent.

60-79: slightly dependent.

40-59: relatively dependent.

20-39: very dependent

Less than 20: completely dependent [27].

The validity and reliability of the Persian version of this tool were confirmed in a study by Tagharrobi et al. [28] The validity of the questionnaire was confirmed using concurrent validity, comparison of known groups and exploratory factor analysis and the reliability was confirmed using Cronbach's alpha. The Cronbach's alpha coefficient of this instrument was reported as 0.99 in this study.

\subsubsection{Quality of life}

To assess the quality of life and the level of social participation (covering the social participation section of the ICF), the EQ-5D-3L questionnaire including 5 questions on a 3-point Likert scale will be used.

The final score of the questionnaire is between 0 to 10 . The validity and reliability of the Persian version were confirmed in a study done by Dastourani et al. [29] The construct validity and the differential validity were confirmed by testing the predetermined hypotheses and by the method of known groups, respectively. Cronbach's alpha coefficient of this questionnaire was reported as 0.89 .

\subsubsection{Depression}

To assess the depression (covering the body structure section of ICF), the BDI-II questionnaire will be used, which includes 21 groups of sentences with a value scale of 0 to 3 expressing the patient's feelings during the previous 2 weeks. The scores of this questionnaire are between 0 and 63 . The validity and reliability of the Persian version of this questionnaire were confirmed in a study done by Hamidi et al. [30] The validity of the questionnaire was confirmed using descriptive and confirmatory factor analysis as well as convergent validity and its Cronbach's alpha was 0.93 .

\subsubsection{Medication adherence}


The MMAS-8 questionnaire will be used to assess patients' medication adherence. This questionnaire consists of 8 questions, of which items 1 to 7 are yes/no questions, and item 8 has a 5-point Likert scale. For items 1 to 7, for each no answer, a score of 1 and for each yes answer, a score of 0 will be recorded. For question 8, scores from 0 to 4 (never, rarely, sometimes, often, always) are considered. The final score will be between 0 and 8 , with 8 indicating high adherence, 6 and 7 indicating moderate adherence, and less than 6 indicating low adherence. The validity and reliability of the Persian version of this questionnaire were confirmed in a study conducted by Ghanei Gheshlagh et al. [31] The validity was confirmed using the concurrent criterion validity method and reliability. Cronbach's alpha coefficient was reported as 0.72 in this study.

\subsubsection{Patient satisfaction}

The PSQ questionnaire will be used to measure patient satisfaction with the treatment. This questionnaire includes 11 items with a 5-point Likert scale (from 0 for very poor to 4 for excellent). The total score is a number between 0 and 44 . The scores of $0-10$ mean poor satisfaction, scores 11-21 mean good satisfaction, scores 22-32 mean very good satisfaction and scores 33-44 mean excellent satisfaction. The validity and reliability of the Persian version of this questionnaire were confirmed in a study done by Yousefi Golafshani et al. [32] The face validity and content validity were confirmed using the opinions of 10 experts in the field of medical education and Cronbach's alpha was reported as 0.98 .

\subsubsection{Complications}

To assess the frequency and type of complications in patients, a stroke complication checklist will be used which includes a total of 19 complications eight of which are based on the most common stroke complications from the perspective of the American Heart Association (AHA) and the American Stroke Association (ASA) [33] and 11 of which are based on the most common complications reported in related studies [34-37]. This checklist is prepared by the researchers and the face and content validity of this checklist will be confirmed by 3 neurologists.

\subsubsection{Data analysis}

Data analysis will be performed using SPSS 22. The data will be first presented in the form of descriptive statistics and frequency distribution tables. The normality of the data will be checked using the Kolmogorov-Smirnov test. In order to determine the relationship between the scores of patients' activities of daily living, quality of life, depression, medication adherence and patient satisfaction with patients' group type (control or intervention), an independent t-test will be used. If there is a significant difference between the intervention group and the control group, the effect of the intervention will be specified. Repeated measures analysis of variance (ANOVA) will also be used to analyze multivariate and control the effects of confounding variables on dependent variables. In all tests, a significance level of 0.05 is considered. Pearson or Spearman correlation coefficient (according to the type of variable distribution) will be used to determine the relationship between the usage duration of program and the score of the measured variables. After categorizing the time of application usage, one-way ANOVA or Kruskal-Wallis test (depending on the type of variable distribution) will be used to determine the relationship between the 
measured variables and the time of application usage. Chi-square test will be used to determine the relationship between the type of complications in both groups. Also, to determine the relationship between the scores of the measured variables before and after the intervention, paired t-test will be used in each group separately. Hypothesis $\mathrm{H} 0$ will indicate the absence of correlation and Hypothesis $\mathrm{H} 1 \mathrm{will}$ indicate the existence of correlation between the measured variables.

\section{Discussion}

Various protocols have been designed to evaluate the impact of mobile applications and trainings on the outcomes of patients with stroke [38-41]. However, these studies differ in terms of sample size, number of variables and outcomes to be measured, duration of intervention and protocol design. In Iran, a study in which first an application is designed using expert and patient needs assessments according to a standard guideline and then the impact of designed application on patients with stroke is evaluated has not been done yet. In other foreign studies, less attention has been paid to different aspects of ICF. However, in the present study, we will try to evaluate the outcomes based on valid and reliable questionnaires that are related to different sections of ICF.

One of the operational problems in such studies is the possibility of patients not being able to work with smartphones or their unwillingness to participate in the study.

Researchers will understand the specific conditions of patients with stroke and will fully explain the purposes of the study to them. If the patient is not able to work with a smartphone, his/her caregiver will be consulted. Of course, this person needs to be someone such as a patient's wife/husband or child who is always available to the patient.

If patients, despite being informed about the goals and efforts of researchers to obtain their satisfaction, seem to be unwilling to participate in the study, according to the principle of informed and free consent, they will be excluded from the study will be replaced by another person with similar conditions.

\section{Trial Status}

Data collection of this study has not yet begun and expected recruitment start date is March to April 2021 and the recruitment will be completed approximately in September 2021.

\section{Declarations}

\section{Ethics approval and consent to participate}

This study was approved in Kashan University of Medical Sciences with the ethics code of IR.KAUMS.NUHEPM.REC.1399.055 and its protocol were registered and approved in the Iranian Registration of Clinical Trial (IRCT) with the code of IRCT20201015049037N1.

- In all stages of conducting research on patients, the neurologist will continuously monitor the method of conducting the study. 
- All usual care, which is performed for patients in the control group, will also be performed for patients in the intervention group, based on the opinion of neurologist.

- Implicit and verbal informed consent will be obtained from all patients and all patients have the right for informed and free consent to participate in the study. Written informed consent will not be obtained due to the possibility of unnecessary sensitivities in patients.

- If a patient decides to stop the intervention in the middle of the research, the researchers consider themselves obliged to respect this request and without changing the treatment, they will exclude the patient from the study and replace this individual with another patient with similar conditions.

- Doing this research will not harm the environment or vulnerable groups.

- Researchers are committed to the principle of privacy and confidentiality and no identity information about patients will be disclosed in the results of the study.

- Researchers are obliged to publish the results of the research honestly, accurately and completely and to provide it to research participants and other stakeholders.

- The method of conducting this study does not contradict any social, cultural and religious values.

The recruitment of this study has not started yet and the expected recruitment start date is March 2021. Results of this study have not already been published or submitted to any journal.

\section{Consent for publication}

Not applicable

\section{Availability of data and materials}

The datasets used and/or analyzed during the current study are available from the corresponding author on reasonable request.

\section{Competing interests}

The authors declare that they have no competing interests.

\section{Funding}

This protocol is done for partial fulfillment of the requirements for the degree of Ph.D. dissertation in the field of health information management, which has been approved by the Research Centre for Health Information Management and the Vice Chancellor for Research of Kashan University of Medical Sciences with the research number 99128 . This internal funder will be provided part or all of the costs for developing application according to the future contract. The authors do not receive any grants from nonprofit organizations or funding agencies either in public or commercial sectors.

\section{Authors' contributions}


HT made substantial contributions to the conception, drafting and design. SAM is the scientific director of the clinical trial and is responsible for assessment of patients. HT, MF, EN are responsible for software development and HA is responsible for recording and analyzing data. All authors have read and approved the manuscript.

\section{Acknowledgements}

Special thanks to Research Centre for Health Information Management and Vice Chancellor for Research and Technology of Kashan University of Medical Sciences for their supports.

\section{Abbreviations}

AHA: American Hospital Association

ANOVA: Analysis of Variance

ASA: American Stroke Association

AU: Attribute of Usability

BDI: Beck Depression Index

Bl: Barthel Index

CVI: Content Validity Index

CVR: Content Validity Ratio

CW: Cognitive Walkthrough

EQ-5D-3L: EuroQol group-5 Dimensions-3 Levels

ICF: International Classification of Functioning, disability and health

MMS-8: Modified Morisky Scale-8 items

NIHSS: National Institute of Health Stroke Scale

PEOU: Perceived Ease of Use

PU: Perceived Usefulness

TAM: Technology Acceptance Model

US: User Satisfaction 


\section{References}

1. Krishnamurthi RV, Feigin VL, Forouzanfar MH, Mensah GA, Connor M, Bennett DA, Moran AE, Sacco $\mathrm{RL}$, Anderson LM, Truelsen T, et al. Global and regional burden of first-ever ischaemic and haemorrhagic stroke during 1990-2010: findings from the Global Burden of Disease Study 2010. The Lancet Global health. 2013;1(5):e259-81.

\section{Update on 2004 Background Paper, BP 6.6 Stroke}

https://www.who.int/medicines/areas/priority_medicines/BP6_6Stroke.pdf.

3. Stroke[MeSH Term] https://www.ncbi.nlm.nih.gov/mesh/68020521.

4. Smith M, Reddy U, Robba C, Sharma D, Citerio G. Acute ischaemic stroke: challenges for the intensivist. Intensive Care Med. 2019;45(9):1177-89.

5. Crichton SL, Bray BD, McKevitt C, Rudd AG, Wolfe CD. Patient outcomes up to 15 years after stroke: survival, disability, quality of life, cognition and mental health. Journal of Neurology Neurosurgery Psychiatry. 2016;87(10):1091-8.

6. Langhorne P, Bernhardt J, Kwakkel G. Stroke rehabilitation. The Lancet. 2011;377(9778):1693-702.

7. Laver KE, Adey-Wakeling Z, Crotty M, Lannin NA, George S, Sherrington C. Telerehabilitation services for stroke. Cochrane Database Syst Rev. 2020;1(1):Cd010255.

8. Anderson K, Burford O, Emmerton L. Mobile Health Apps to Facilitate Self-Care: A Qualitative Study of User Experiences. PloS one. 2016;11(5):e0156164.

9. Kamal AK, Shaikh Q, Pasha O, Azam I, Islam M, Memon AA, Rehman H, Akram MA, Affan M, Nazir S, et al. A randomized controlled behavioral intervention trial to improve medication adherence in adult stroke patients with prescription tailored Short Messaging Service (SMS)-SMS4Stroke study. BMC Neurol. 2015;15(1):212.

10. Guidetti S, Gustavsson M, Tham K, Andersson M, Fors U, Ytterberg C. F@ce: a team-based, personcentred intervention for rehabilitation after stroke supported by information and communication technology - a feasibility study. BMC Neurol. 2020;20(1):387.

11. Shin $\mathrm{DC}$, Song $\mathrm{CH}$. Smartphone-based visual feedback trunk control training using a gyroscope and mirroring technology for stroke patients: single-blinded, randomized clinical trial of efficacy and feasibility. American journal of physical medicine rehabilitation. 2016;95(5):319-29.

12. Kim DY, Kwon H, Nam KW, Lee Y, Kwon HM, Chung YS. Remote Management of Poststroke Patients With a Smartphone-Based Management System Integrated in Clinical Care: Prospective, Nonrandomized, Interventional Study. J Med Internet Res. 2020;22(2):e15377.

13. Linder SM, Rosenfeldt AB, Bay RC, Sahu K, Wolf SL, Alberts JL. Improving Quality of Life and Depression After Stroke Through Telerehabilitation. The American journal of occupational therapy: official publication of the American Occupational Therapy Association. 2015;69(2):6902290020p6902290021-6902290010.

14. Des Roches CA, Balachandran I, Ascenso EM, Tripodis Y, Kiran S. Effectiveness of an impairmentbased individualized rehabilitation program using an iPad-based software platform. Front Hum 
Neurosci. 2014;8:1015.

15. Mayo NE, Nadeau L, Ahmed S, White C, Grad R, Huang A, Yaffe MJ, Wood-Dauphinee S. Bridging the gap: the effectiveness of teaming a stroke coordinator with patient's personal physician on the outcome of stroke. Age Ageing. 2008;37(1):32-8.

16. Huijgen BC, Vollenbroek-Hutten MM, Zampolini M, Opisso E, Bernabeu M, Van Nieuwenhoven J, Ilsbroukx S, Magni R, Giacomozzi C, Marcellari V, et al. Feasibility of a home-based telerehabilitation system compared to usual care: arm/hand function in patients with stroke, traumatic brain injury and multiple sclerosis. J Telemed Telecare. 2008;14(5):249-56.

17. Chávez A, Borrego G, Gutierrez-Garcia JO, Rodríguez LF. Design and evaluation of a mobile application for monitoring patients with Alzheimer's disease: A day center case study. International journal of medical informatics. 2019;131:103972.

18. Moral-Munoz JA, Zhang W, Cobo MJ, Herrera-Viedma E, Kaber DB. Smartphone-based systems for physical rehabilitation applications: A systematic review. Assistive Technology 2019:1-14.

19. Piran P, Thomas J, Kunnakkat S, Pandey A, Gilles N, Weingast S, Burton D, Balucani C, Levine SR. Medical Mobile Applications for Stroke Survivors and Caregivers. Journal of Stroke Cerebrovascular Diseases. 2019;28(11):104318.

20. Zhou X, Du M, Zhou L. Use of mobile applications in post-stroke rehabilitation: a systematic review. Topics in stroke rehabilitation 2018:1-11.

21. Parke HL, Epiphaniou E, Pearce G, Taylor SJC, Sheikh A, Griffiths CJ, Greenhalgh T, Pinnock H. SelfManagement Support Interventions for Stroke Survivors: A Systematic Meta-Review. PloS one. 2015;10(7):e0131448-8.

22. Miranda JJ, Moscoso MG, Toyama M, Cavero V, Diez-Canseco F, Ovbiagele B. Role of mHealth in overcoming the occurrence of post-stroke depression. Acta Neurol Scand. 2018;137(1):12-9.

23. Sureshkumar K, Murthy GV, Munuswamy S, Goenka S, Kuper H. 'Care for Stroke', a web-based, smartphone-enabled educational intervention for management of physical disabilities following stroke: feasibility in the Indian context. BMJ innovations. 2015;1(3):127-36.

24. Polson PG, Lewis C, Rieman J, Wharton C. Cognitive walkthroughs: a method for theory-based evaluation of user interfaces. International Journal of man-machine studies. 1992;36(5):741-73.

25. Choi YH, Ku J, Lim H, Kim YH, Paik NJ. Mobile game-based virtual reality rehabilitation program for upper limb dysfunction after ischemic stroke. Restor Neurol Neurosci. 2016;34(3):455-63.

26. Hinkle JL. Reliability and validity of the National Institutes of Health Stroke Scale for neuroscience nurses. Stroke. 2014;45(3):e32-4.

27. Mahoney FI, Barthel DW. Functional evaluation: the Barthel Index: a simple index of independence useful in scoring improvement in the rehabilitation of the chronically ill. Maryland state medical journal 1965.

28. Tagharrobi Z, Sharifi K, Sooky Z. Psychometric evaluation of Shah version of modified Barthel index in elderly people residing in Kashan Golabchi nursing home. KAUMS Journal (FEYZ). $2011 ; 15(3): 213-24$. 
29. Dastourani A, Mansour Sohani S, Shah Ali S. Reliability and validity of the Persian version of the european quality of life questionnaire (EQ-5D-3L) in patients with meniscus and knee ligaments injury. Journal of Paramedical Sciences Rehabilitation. 2018;7(4):73-82.

30. Hamidi R. Validity and reliability Beck depression inventory-II among the Iranian elderly population. 2015.

31. Ghanei Gheshlagh R, Ebadi A, Veisi Raygani A, Nourozi Tabrizi K, Dalvandi A, Mahmoodi H. Determining concurrent validity of the Morisky medication adherence scale in patients with type 2 diabetes. Iranian Journal of Rehabilitation Research. 2015;1(3):24-32.

32. Tabatabaee SM. Evaluation of validity and reliability of patient satisfaction questionnaire in Firoozgar Hospital clinic. Razi Journal of Medical Sciences. 2016;22(139):121-7.

\section{3. let's talk about Complications After Stroke [https://.}

34. Rohweder G, Ellekjær H, Salvesen $\varnothing$, Naalsund E, Indredavik B. Functional outcome after common poststroke complications occurring in the first 90 days. Stroke. 2015;46(1):65-70.

35. Brown DL, Smith TL, Johnston KC: Chap. 17 Medical Complications of Stroke. In: Blue Books of Practical Neurology. Volume 29, edn. Edited by Kasner SE, Gorelick PB: Butterworth-Heinemann; 2004: 349-362.

36. Zorowitz RD, Tietjen GE. Medical complications after stroke. Journal of Stroke Cerebrovascular Diseases. 1999;8(3):192-6.

37. Kumar S, Selim MH, Caplan LR. Medical complications after stroke. Lancet Neurol. 2010;9(1):10518.

38. Koh GC-H, Yen SC, Tay A, Cheong A, Ng YS, De Silva DA, Png C, Caves K, Koh K, Kumar Y. Singapore Tele-technology Aided Rehabilitation in Stroke (STARS) trial: protocol of a randomized clinical trial on tele-rehabilitation for stroke patients. BMC Neurol. 2015;15(1):1-14.

39. Vansimaeys C, Zuber M, Pitrat B, Join-Lambert C, Tamazyan R, Farhat W, Bungener C. Combining standard conventional measures and ecological momentary assessment of depression, anxiety and coping using smartphone application in minor stroke population: a longitudinal study protocol. Frontiers in psychology. 2017;8:1172.

40. Gauthier LV, Kane C, Borstad A, Strahl N, Uswatte G, Taub E, Morris D, Hall A, Arakelian M, Mark V. Video Game Rehabilitation for Outpatient Stroke (VIGoROUS): protocol for a multi-center comparative effectiveness trial of in-home gamified constraint-induced movement therapy for rehabilitation of chronic upper extremity hemiparesis. BMC Neurol. 2017;17(1):109.

41. Kamal AK, Shaikh QN, Pasha O, Azam I, Islam M, Memon AA, Rehman H, Affan M, Nazir S, Aziz S, et al. Improving medication adherence in stroke patients through Short Text Messages (SMS4Stroke)study protocol for a randomized, controlled trial. BMC Neurol. 2015;15(1):157.

\section{Figures}




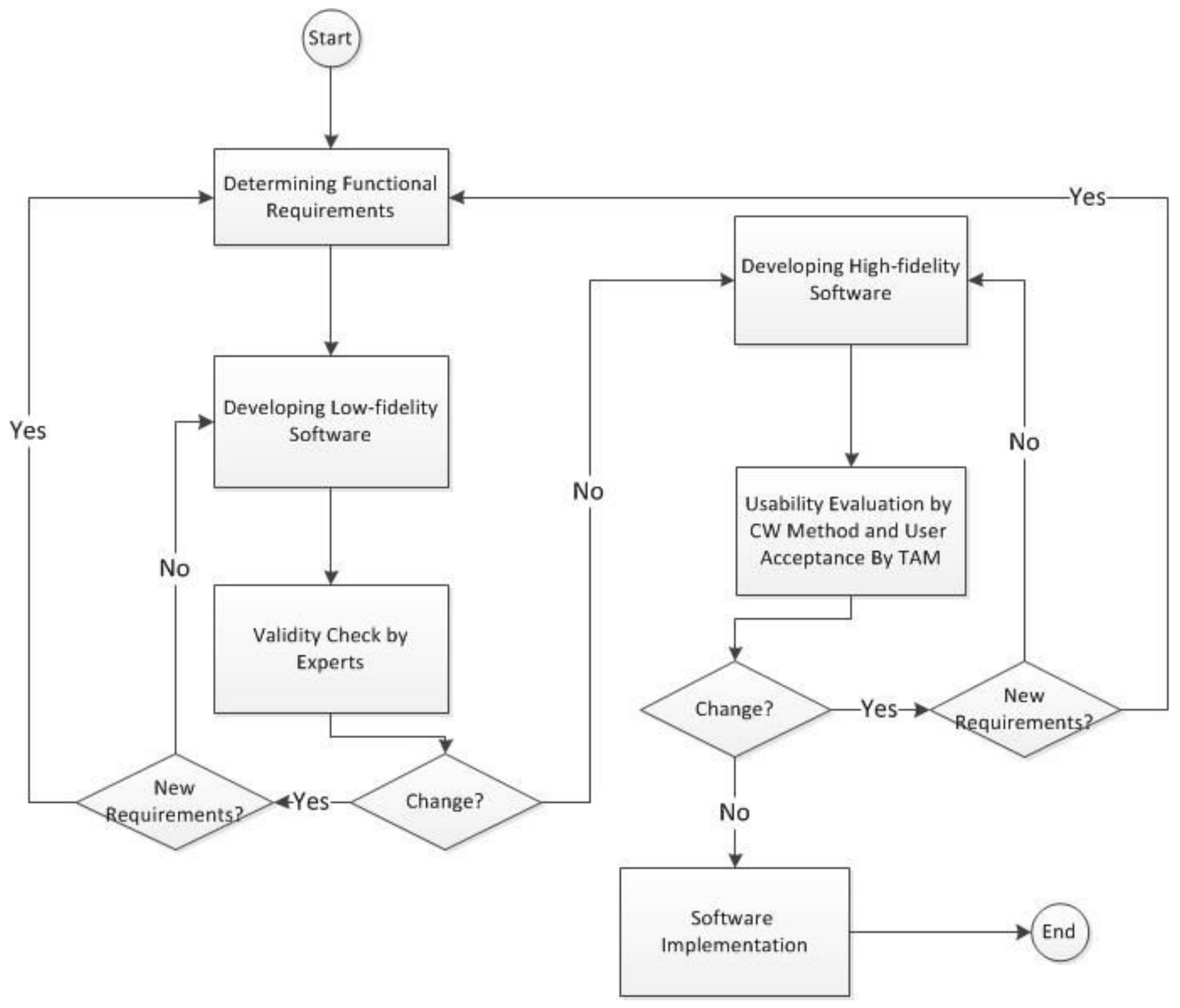

Figure 1

Method of Software Development 


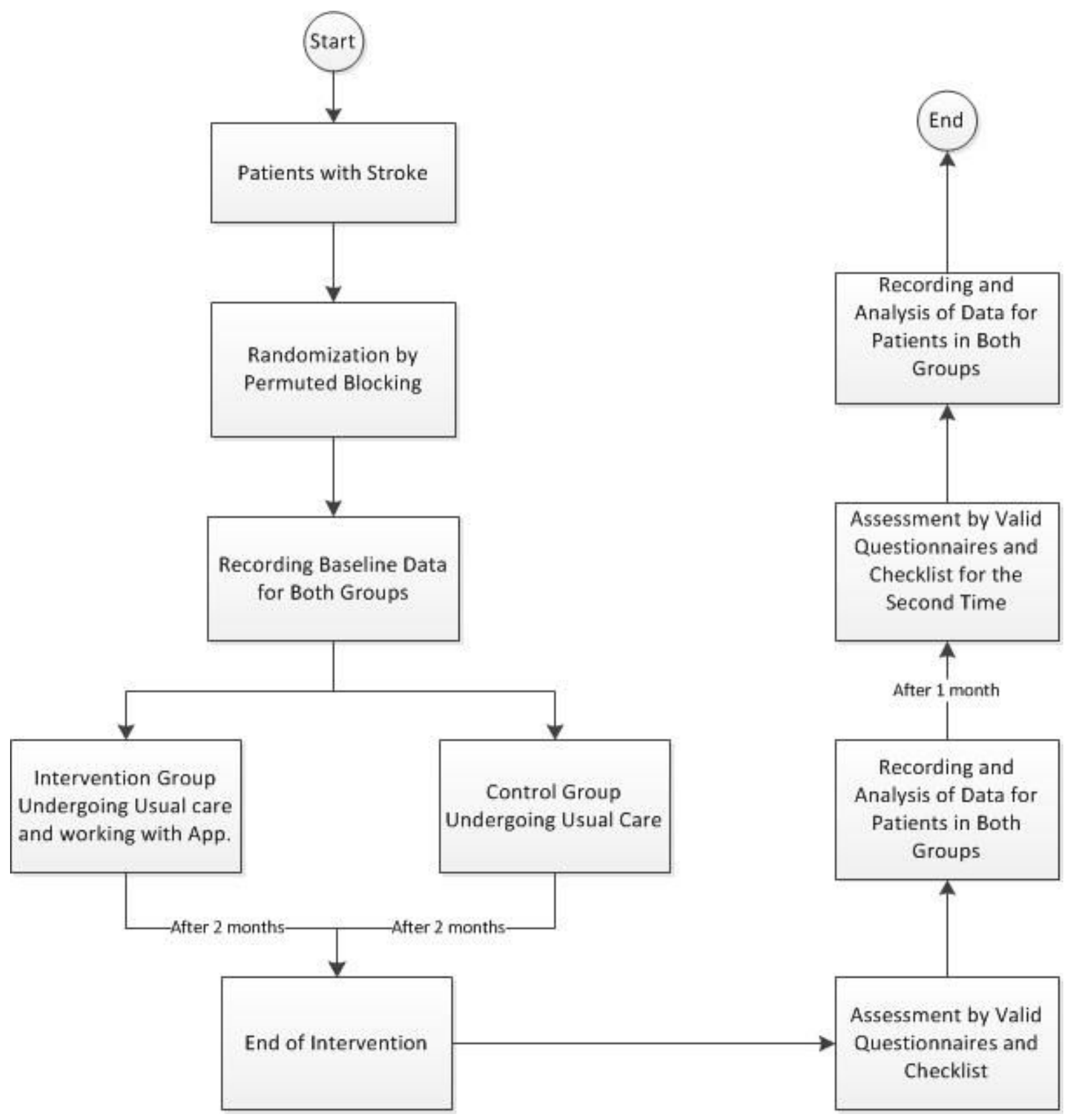

\section{Figure 2}

Protocol for Interventional Trial

\section{Supplementary Files}

This is a list of supplementary files associated with this preprint. Click to download.

- SPIRITchecklist.docx 